\title{
TemplateFlow: a community archive of imaging templates and atlases for improved consistency in neuroimaging
}

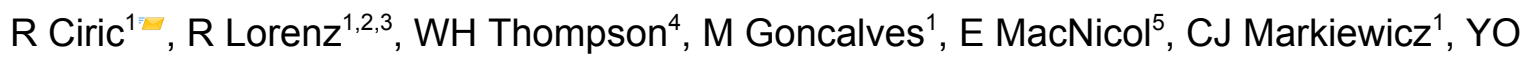
Halchenko $^{6}$, SS Ghosh ${ }^{7,8}$, KJ Gorgolewski $^{1}$, RA Poldrack ${ }^{1}$, and O Esteban ${ }^{1,9}$

${ }^{1}$ Department of Psychology, Stanford University, CA, USA; ${ }^{2} \mathrm{MRC}$ CBU, University of Cambridge, Cambridge, UK; ${ }^{3}$ Department of Neurophysics, MPI, Leipzig, Germany; ${ }^{4}$ Department of Clinical Neuroscience, Karolinska Institutet, Stockholm, Sweden; ${ }^{5}$ Department of Neuroimaging, Institute of Psychiatry, Psychology and Neuroscience, King's College London, London, UK; ${ }^{6}$ Department of Psychological and Brain Sciences, Dartmouth College, Hanover, $\mathrm{NH}$, USA. ${ }^{7}$ McGovern Institute for Brain Research, Massachusetts Institute of Technology: MIT, Cambridge, MA, USA; ${ }^{8}$ Department of Otolaryngology, Harvard Medical School, Boston, MA, USA; ' ${ }^{2}$ Department of Radiology, University Hospital of Lausanne and University of Lausanne, Lausanne, Switzerland.

Correspondence: RC <rastko@stanford.edu>, OE <phd@oscaresteban.es>

Neuroimaging templates and corresponding atlases play a central role in experimental workflows and are the foundation for reporting standardised results. The proliferation of templates and atlases is one relevant source of methodological variability across studies, which has been recently brought to attention as an important challenge to reproducibility in neuroscience. Unclear nomenclature, an overabundance of template variants and options, inadequate provenance tracking and maintenance, and poor concordance between atlases introduce further unreliability into reported results. We introduce Templateflow, a cloud-based repository of human and nonhuman imaging templates paired with a client application for programmatically accessing resources. TemplateFlow is designed to be extensible, providing a transparent pathway for researchers to contribute and vet templates and their associated atlases. Following software engineering best practices, TemplateFlow leverages technologies for unambiguous resource identification, data management, versioning and synchronisation, programmatic extensibility, and continuous integration. By equipping researchers with a robust resource for using and evaluating templates, TemplateFlow will contribute to increasing the reliability of neuroimaging results.

\section{Introduction}

Brains are morphologically variable, exhibiting diversity in such features as overall size ${ }^{1}$, sulcal curvature ${ }^{2}$, and functional topology $y^{3,4}$. Morphological variability manifests not only in differences between brains but also in the way that a brain changes across its lifespan, as it is remodelled by development, ageing, and degenerative processes ${ }^{5-7}$. These morphological 
differences often correspond with the effects of interest in neuroimaging studies and hinder direct spatial comparisons between brain maps ${ }^{8}$. The substantial variability within and between individual brains necessitates a means of formalising population-level knowledge about brain anatomy and function. Neuroscientists have answered this need by creating brain atlases as references for understanding and contextualising morphological variability. Atlases encapsulate landmarks, features, and other kinds of knowledge about the brain as annotations that are consistent across individual brains.

The development of atlases in neuroscience has accelerated knowledge discovery and dissemination. Early endeavours, epitomised by the groundbreaking work of Brodmann, leveraged careful scrutiny of microanatomy and cytoarchitectonic properties in small numbers of brains $^{9,10}$. Concurrent macroanatomical approaches, by contrast, identified common features in nuclear boundaries and cortical gyrification. Modern atlases advanced on these approaches by incorporating stereotaxy ${ }^{11}$, defining a basis set of coordinate axes over the brain and anchoring neural landmarks to coordinates. Initially developed over a century ago to surgically induce targeted brain lesions, the first stereotaxic apparatus informed early sectional atlases of the cat and macaque brains ${ }^{11}$. In humans, Talairach's assiduous postmortem examination of a single human brain produced a stereotaxic atlas that saw wide use ${ }^{12}$. Since then, neuroscientists have directed great efforts to improve existing ${ }^{13}$ and generate new atlases of the neurotypical adult human $^{14}$ and nonhuman ${ }^{15,16}$ brain; as well as developing, ageing, and neurologically atypical brains. For instance, new atlases and representative stereotaxic maps can be created for diseased $^{17}$, infant $^{18-20}$, and elderly ${ }^{21}$ human populations or to capture the rapid postnatal development of nonhuman species ${ }^{22,23}$. Recently, atlasing endeavours have largely shifted away from the search for a single universal neuroanatomical pattern, instead making use of increasingly large samples with the aim of representing a population average of the distribution of morphological patterns.

On account of its relatively high spatial resolution, its capacity to image the entire brain, and its non-invasive acquisition protocols, magnetic resonance imaging (MRI) has revolutionised neuroscience in general and the atlasing endeavour ${ }^{24}$ in particular. For three cardinal reasons, atlases have become an indispensable component of modern neuroimaging data workflows. First, group inference in neuroimaging studies requires that individuals' features are aligned into a common spatial frame of reference where their location can be called standard ${ }^{8}$. Second, software instruments' progress to map homologous features between subjects ${ }^{25,26}$ has enabled researchers to create population-average maps of a particular image modality with relative ease using commonly available software. These maps, called templates, are typically created by averaging images that are representative of the population of interest to a study ${ }^{27,28}$. Concomitant advances in image acquisition, processing, and analysis have enabled new templates to build iteratively upon previous work $^{24}$. Third, templates engender a stereotaxic coordinate system in which atlases can be delineated or projected. Associating atlases with template coordinates also facilitates the mapping of prior population-level knowledge about the brain into images of individual subjects' brains (for instance, to sample and average the functional MRI signal indexed by the regions defined in an atlas ${ }^{29}$ ).

Because they are integral to analytic workflows and because atlasing technology is continuously improving, a multiplicity of brain templates and atlases have been published ${ }^{28}$. Factors that have stimulated the proliferation of templates include distribution within software toolboxes, data structuring conventions, maintenance, and issues with licensing and shareability. As a result, researchers have at their disposal a wealth of open-access templates and atlases as well as established protocols for the creation of study-specific alternatives. Recent research ${ }^{30}$ has cautioned that a naive more-is-better assumption for methodological 
options lends itself to greater methodological flexibility, which can threaten the reproducibility of findings. This problem is evidently not new for the neuroimaging community ${ }^{31}$, and the proliferation of templates and atlases adds on to the methodological degrees of freedom available to researchers. In the particular case of templates and atlases, the problem is exacerbated by poor consistency across available alternatives, as exposed by Bohland et al. ${ }^{32}$. Along similar lines, Yoon et al. cautioned about "template effects" confounding the interpretation of results from pediatric imaging studies based on a common adult reference template ${ }^{27}$. The concerning picture is completed with inaccurate reporting ${ }^{33}$, as it is often difficult (if not impossible) to map reports in literature back to the actual template or atlas employed in the report. Most prominently, the ubiquitous reporting of results (e.g., peak coordinates) "in the MNI (Montreal Neurological Institute) standard space" is inadequate because there is a large portfolio of templates ${ }^{6,19,24,34}$ developed and distributed by the MNI. Moreover, the widespread FSL toolbox ${ }^{35}$ references its results to an "MNI space" neither officially created nor distributed by the MNI.

To address the need for a centralized resource for the archiving and redistribution of templates and atlases that allows programmatic access to human and nonhuman imaging templates ${ }^{36}$, we have developed TEMPLATEFLow (Figure 1). The resource is envisioned to support the emergence of processing and analysis workflows ${ }^{37,38}$ that brain mapping is witnessing, while addressing the above concerns threatening reproducibility. TEMPLATEFLow is modular, and both data and software are version-controlled. The resource allows researchers to use templates "off-the-shelf" and share new ones. An online documentation hub provides further details and facilitates use (http://www.templateflow.org). 

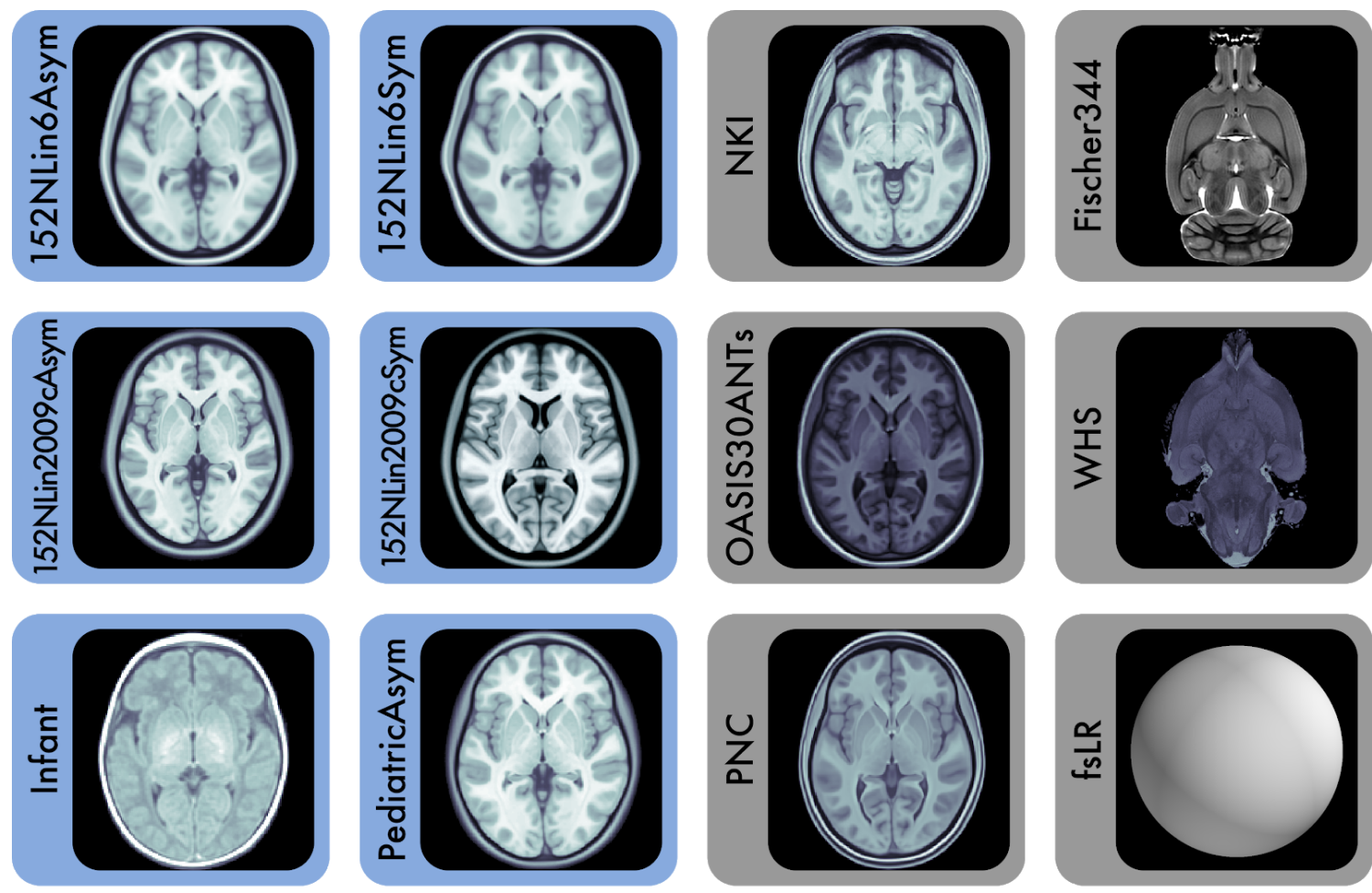

MNI templates
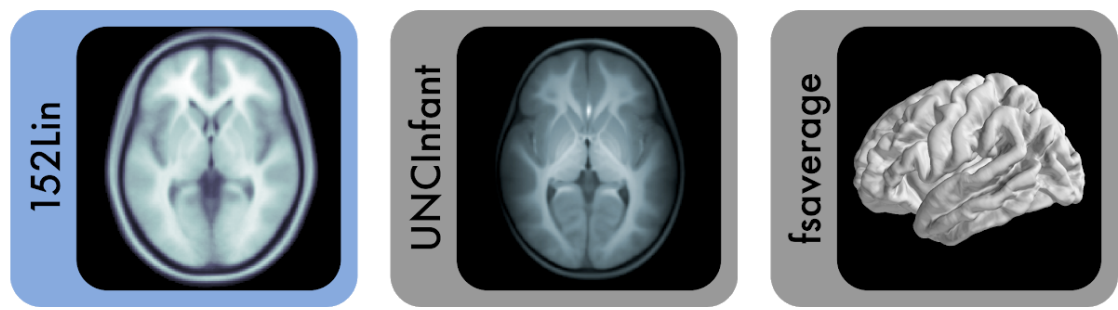

Figure 1. Representative views of 15 templates currently available in the TempLATEFLow archive. The 7 templates highlighted in blue are constituents of the Montreal Neurological Institute (MNI) portfolio. The Waxholm space (WHS) and Fischer344 templates provide references for rat neuroimaging. fsaverage and fs $L R$ are surface templates; the remaining templates are volumetric. Each template is distributed with atlas labels, segmentations, and metadata files. The 15 templates displayed here are only a small fraction of those created as stereotaxic references for the neuroimaging community.

\section{Results}

The ambiguity of MNI space reporting, and the case for a centralised repository with uniform nomenclature.

The lack of consistent template nomenclature introduces ambiguity in scientific communication, which is further biased by a researcher's choice of software library. Perhaps most notably, the majority of neuroimaging literature reports results spatially normalized and given in the Montreal Neurological Institute (MNI) standard coordinates. However, this statement is imprecise, as MNI offers a wide portfolio of MRI templates ${ }^{19,24,34,39-42}$. Although all these "MNI 
templates" are spatially aligned to the "MNI Average Brain (305 MRI) Stereotaxic Registration Model $^{41 "}$ or just $M N I 305$, leading neuroimaging toolboxes are not consistent in their default choice of MNI template.

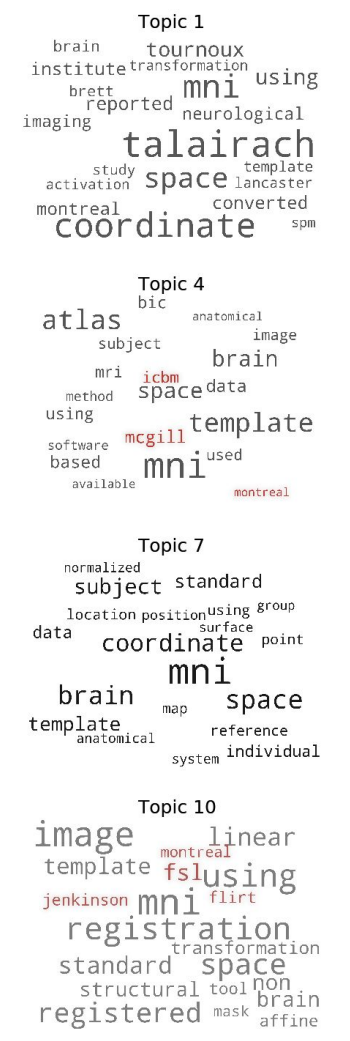

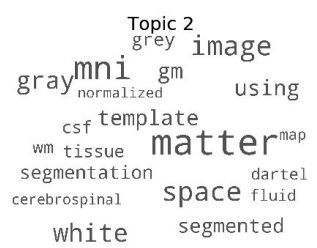

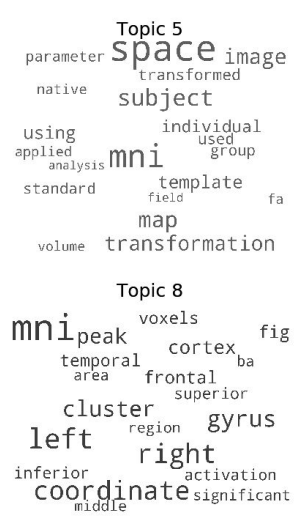

kernel ${ }^{\text {Topic } 11}$ space

template isotropic $\mathrm{mm}$ maximum full
age montreal
gaussian image gaussian fwh institute thed $\mathrm{mni}$ normalized using

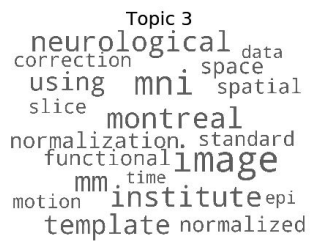

template normalized

ucl image mapping using brain volume mni Spm london mri slice space wellcome slita template
weighted normalized software Topic 9 instituteusing brain based mni montreal source vertemplate mesh neurological model $1^{x y z}$ surface

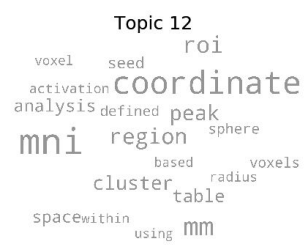

Figure 2. Word cloud visualisations of a topic model of sentences from scientific articles that reference MNI space. For each of 12 topics identified by the model, the 20 words with the highest loadings on that topic are displayed. A larger font size indicates that a word loads more strongly on the topic. When qualitatively inspecting the topics, we identified three that provided insight into template provenance: topic 4 (MNI/ICBM), topic 6 (SPMMellcome Centre), and topic 10 (FSL/FMRIB). Provenance-related words are highlighted for these topics.

To investigate the heterogeneous use of MNI templates in the neuroimaging literature, we performed an exploratory text mining analysis. Across the entire corpus of articles published in two leading methodological journals (Neurolmage and Neurolmage: Clinical), we identified 6,048 articles containing 14,870 sentences that included the term MNI. After preprocessing the sentence text, we used latent Dirichlet allocation ${ }^{43}$ to create a topic model of the surveyed articles. A qualitative inspection of topic-word loadings suggested that of the 12 topics we identified, three provided insight into the likely provenance of the templates that an article used (Figure 2). Two of these corresponded to two software packages widely used in neuroimaging - SPM (topic 6) and FSL (topic 10)-each of which is distributed with particular versions of the MNI template. The third (topic 4) related to the International Consortium for Brain Mapping (ICBM) and the MNI itself, the institutions that oversee the creation and curation of the MNI template portfolio. 


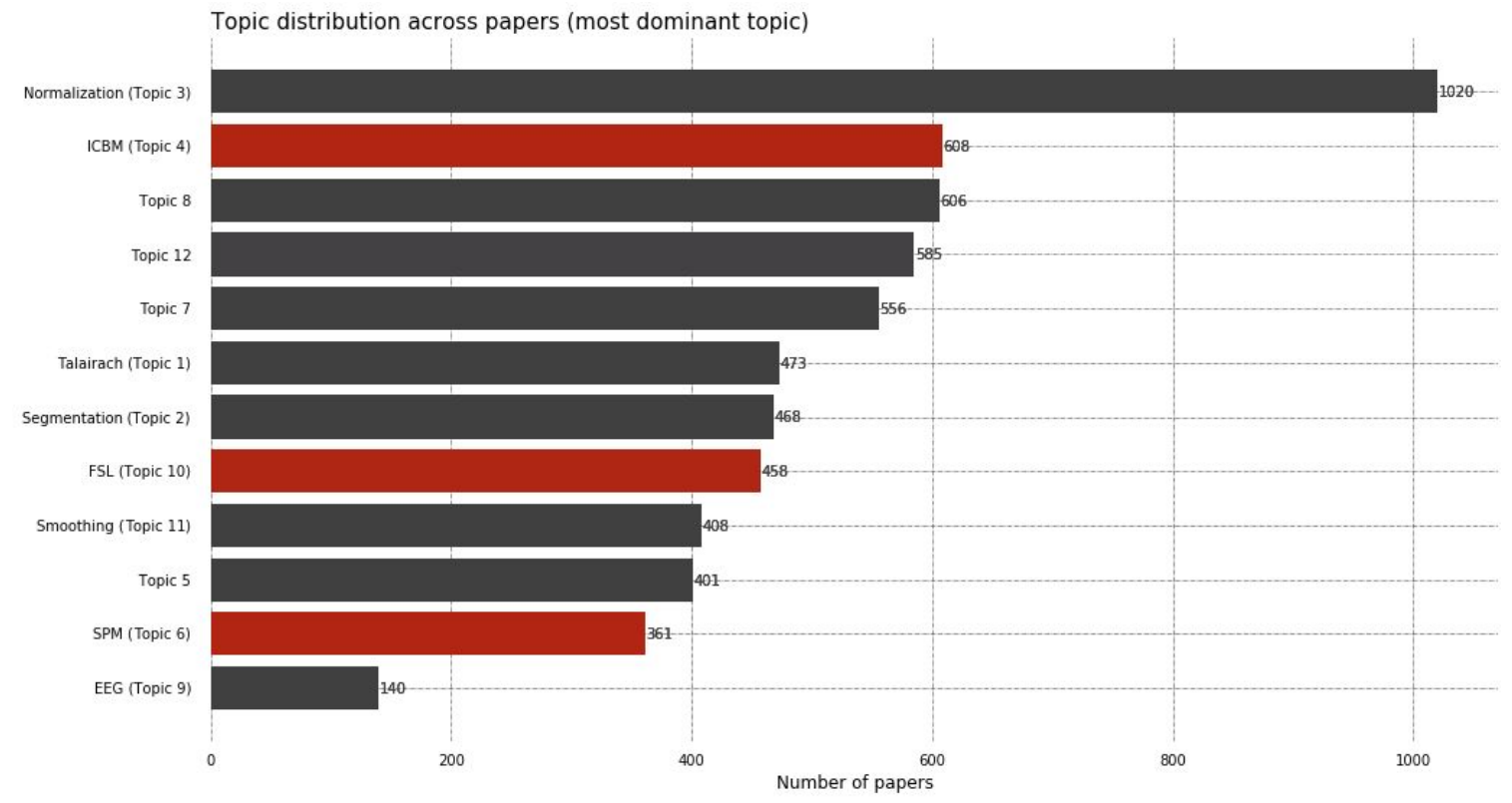

Figure 3. Topic distribution across articles. Each bar indicates the number of surveyed articles in which a topic was identified as dominant. The dominant MNI-related topic in a plurality of surveyed articles was related to general normalisation and registration. Topics corresponding to template provenance (topic 4: ICBM, topic 6: SPM, and topic 10: FSL) were each dominantly present in hundreds of articles, underscoring the heterogeneity of MNI templates used in the literature. The absence of provenance-related terms in many other articles showcases the potential difficulties in determining the template resources that were used in a neuroimaging study.

To demonstrate the heterogeneity of MNI template usage, we sorted articles according to their dominant topic (i.e., the topic with the highest model score in MNI-related sentences; Figure 3). We found that hundreds of articles featured each of the three provenance-related topics we had previously identified, underscoring that "MNI template" can refer to any of a family of templates and is not a unique identifier. As a matter of fact, studies carried out with SPM96 ${ }^{44}$ and earlier versions report their results "in MNI space" with reference to the single-subject Colin 27 average template ${ }^{42}$. However, beginning with SPM99, SPM updated its definition of "MNI space" to the template that MNI released in 2001: an average of 152 subjects from the ICBM database, aligned by means of linear registration. In SPM12 (the latest release at the time of writing), the meaning of "MNI" varies by submodule: different modules alternately use the Linear MNI152 template and a new, nonlinear revision from 2009. By contrast, the "MNI space" template bundled with the FSL toolbox was developed by Dr. A. Janke in collaboration with MNI researchers ${ }^{24}$. Although it was generated under the guidance of and using the techniques of the 2006 release of nonlinear MNI templates, this template is not in fact part of the official portfolio distributed by MNI. Nonetheless, our results suggest that the MNI templates bundled with SPM and FSL have historically gained broader currency as a result of the widespread use of these software libraries. 
Although our results are only a first approximation, and although they do not provide insight into the provenance of the majority of MNI templates, they present several important cases for consideration. First, absent an unambiguous reporting nomenclature (such as Research Resource Identifiers ${ }^{45}$; RRIDs), the widespread use of ambiguous terms like "MNI" presents a potential barrier for reproducing results and increases the chance of misapplying coordinates or references from an incorrect space. Second, absent a readily accessible centralised repository, researchers might often default to templates that are easy to access, many of which are tied to specific software packages. Third, as illustrated by the changing definition of "MNI" in SPM, template references can change as new technologies emerge, suggesting an essential need for version control systems. Finally, our analysis illustrates a fourth requirement for unambiguous reporting of results: a consensus regarding the minimally sufficient provenance-related information to report in studies and to distribute with templates. We sought to develop TEMPLATEFLow with these features in mind.

\section{A version-controlled archive of neuroimaging templates maximizing the accuracy in reporting spatially standardized results.}

TEMPLATEFLow is a cloud-based repository of human and nonhuman imaging templates paired with a Python-based client for programmatically accessing template resources. TemplateFlow addresses the need for a standard, centralised repository of templates and corresponding atlases and metadata. The TEMPLATEFLow ARCH IVE has a tree-directory structure, metadata files, and data files following a standard inspired by the Brain Imaging Data Structure ${ }^{46}$ (BIDS). BIDS is a widespread standard that balances the needs for human- and machine-readability. BIDS prescribes a file naming scheme comprising a series of key-value pairs (called "entities") that are ordered hierarchically.

The most salient entity is the template identifier (signified with the key tpl-), whose value is an alphanumeric label that is unique across the ARCHIve (e.g., tpl-MNI152Lin). Table 1 enumerates several templates currently distributed with the ARCHIVE, and their corresponding unique identifiers. The unique identifier resolves the issue of inaccurate reporting, as it unambiguously designates one specific template. In addition, because the repository is versioned, researchers can easily retrieve and report the exact version of the template or atlas that was used in their study. Supplementary Table S1 summarizes the available entities and shows a segment of the file organization of the ARCHIVE. For each template, the TEMPLATEFLow database includes one or more reference volumetric template images (e.g., one T1-weighted and one T2-weighted average map; all must be in register), a set of atlas labels and voxelwise annotations defined with reference to the template image, and additional files containing the template and atlas metadata. Figure 4 summarizes the data types and metadata that can be stored in the ARCHIVE. Figure 5 provides an overview of the ARCHIVE's metadata specification.

Cloud storage for the ARCHIVE is supported by the Open Science Framework (osf.io) and Amazon's Simple Storage Service (S3). Version control, replication, and synchronisation of template resources across filesystems is managed with $\mathrm{D}_{\mathrm{ATALAD}}{ }^{47}$.

Table 1. Digital templates included in TEMPLATEFLow. TEMPLATEFLOW is designed to maximise the discoverability and accessibility of new templates, minimise redundancies in 
template creation, and promote standardisation of processing workflows. To enhance visibility of existing templates, TEMPLATEFLOW includes a web-based browser indexing all files in the TEMPLATEFLOW ARCHIVE (templateflow.org/browse/).

\begin{tabular}{|c|c|}
\hline Template (Unique ID) & Description \\
\hline MNI152Lin Li, $39^{39}$ & $\begin{array}{l}\text { Neurotypical adult human template created as the average from a } \\
\text { linear mapping of } 152 \text { subjects from the MNI cohort of the ICBM } \\
\text { registered to the earlier MNI } 305 \text { template. }\end{array}$ \\
\hline
\end{tabular}

MNI152NLin6Asym ${ }^{24} \quad$ FSL's version of the MNI152 neurotypical adult human template created using iterative nonlinear registration and averaging.

MNI152NLin6Sym ${ }^{24} \quad$ Symmetric version of MNI152NLin6Asym.

MNI152NLin2009 CAsym $^{19,40}$ Update of the MNI152 neurotypical adult template with nonlinear registration. The mapping and averaging proceeded over 40 iterations beginning from the earlier MNI152 template.

MNI152NLin2009cSym ${ }^{19,40}$ Symmetric version of MNI152NLin2009cAsym.

MNIInfant $^{19}$
Series of human infant templates created from 11 cohorts of infants
and young children. Each cohort spans a different age range
between 0 and 60 months.

MNIPediatricAsym ${ }^{19,40}$

Series of human pediatric templates created from 6 partially overlapping cohorts of children and young adults. Each cohort spans a different age range between 4.5 and 18.5 years.

\begin{tabular}{|ll}
\hline NKI $^{14,48}$ & $\begin{array}{l}\text { Template created for the NKI-Rockland sample using ANTs } \\
\text { diffeomorphic registration and averaging. }\end{array}$ \\
\hline OASIS30ANTs ${ }^{14,49}$ & $\begin{array}{l}\text { Template created using ANTs diffeomorphic registration and } \\
\text { averaging for the Open Access Series of Imaging Studies (OAS }\end{array}$ \\
\hline PNC $^{50}$ & $\begin{array}{l}\text { Pediatric and young adult template created using ANTs } \\
\text { diffeomorphic registration and averaging for the Philadelphia } \\
\text { Neurodevelopmental Cohort. }\end{array}$ \\
\hline UNCInfant $^{2 \theta}$ & $\begin{array}{l}\text { Series of human infant templates created from a 95-subject } \\
\text { longitudinal sample comprising three scans: as neonates, as } \\
\text { one-year-olds, and as two-year-olds. }\end{array}$
\end{tabular}

WHS $^{51-54} \quad$ Waxholm space template created as an atlas of the Sprague-Dawley rat brain.

Fischer $344^{55}$

$f S L R^{56}$

fsaverage ${ }^{57}$
Rat template created as the average of 41 four-month-old animals from the Fischer 344 strain.

Surface-based Freesurfer template created for the Human Connectome Project (HCP).

Surface-based average Freesurfer template. 


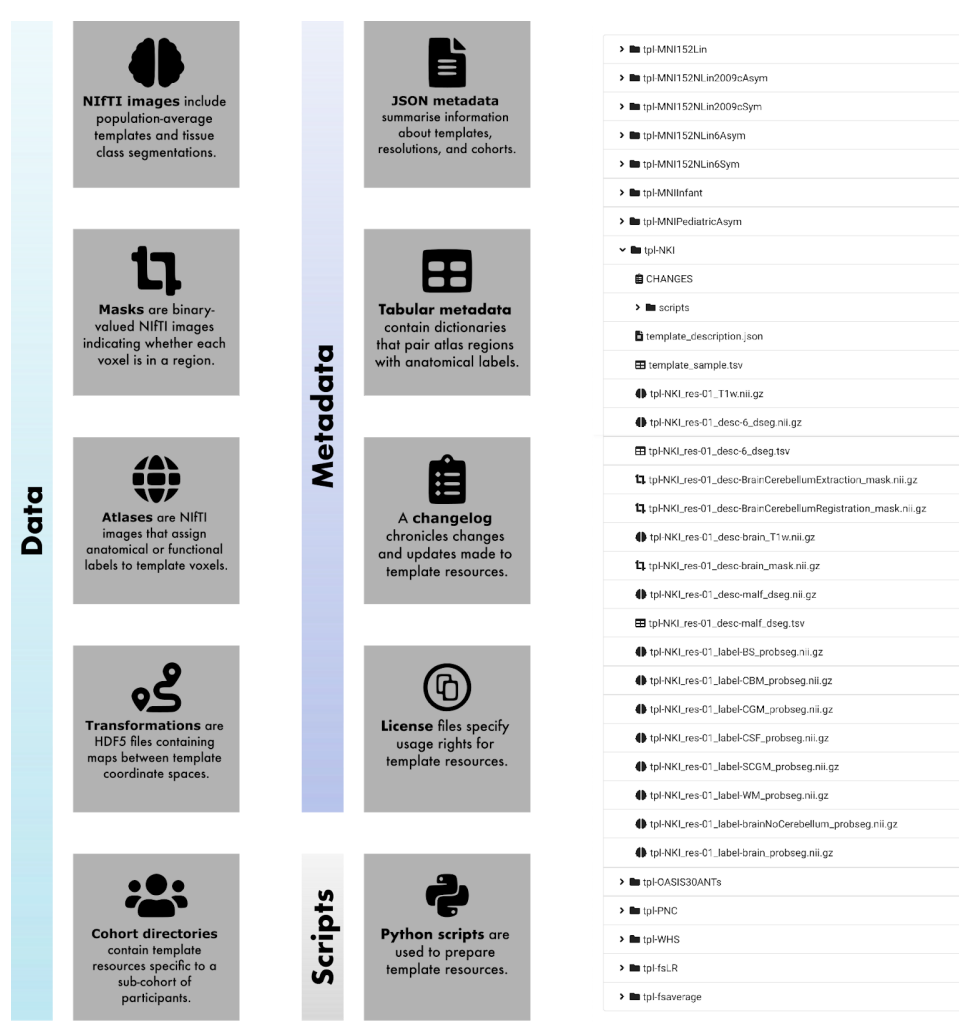

Figure 4. The TEMPLATEFLOW ARCHIVE contains template resources. Left, common file formats included in the TEMPLATEFLOW ARCHIVE. Right, view of the TEMPLATEFLOW ARCHIVE browser, accessible from templateflow.org, with a single template resource directory expanded. Template data are archived using a BIDS-like directory structure, with top-level directories for each template. Each directory contains image files, annotations, and metadata for that template. Following BIDS specifications, volumetric data are stored in NIfTI format and surface data with the GIFTI2 format.

\section{A key neuroimaging resource developed with the best software engineering standards and easily operable by machines.}

TEMPLATEFLow's Python client provides human users and software tools with reliable and programmatic access to the archive. The client can be integrated seamlessly into image processing workflows to handle requests for template resources on the fly. It features an intuitive application programming interface (API) that can query the TEMPLATEFLow ARCHIVE for specific files (Figure 6). The BIDS-inspired organization enables easy integration of tools and infrastructure designed for BIDS (e.g., the Python client uses PyBIDS ${ }^{58}$ to implement the queries listed in Table S1). To query TempLAteFLow, a user can submit a list of arguments corresponding to the BIDS-like key-value pairs in each entity's file name (e.g., atlas=Schaefer2018 to return files containing voxelwise annotations from the 2018 Schaefer atlas ${ }^{59}$ ).

To integrate template resources into neuroimaging workflows, traditional approaches required deploying an oftentimes voluminous tree of prepackaged data to the filesystem. By contrast, the TEMPLATEFLow client implements lazy loading, which permits the base installation to be extremely lightweight. Instead of distributing neuroimaging data with the installation, TemplateFlow allows the user to dynamically pull from the cloud-based storage only those resources they need, as they need them. After a resource has been requested once, it remains cached in the filesystem for future utilization. 


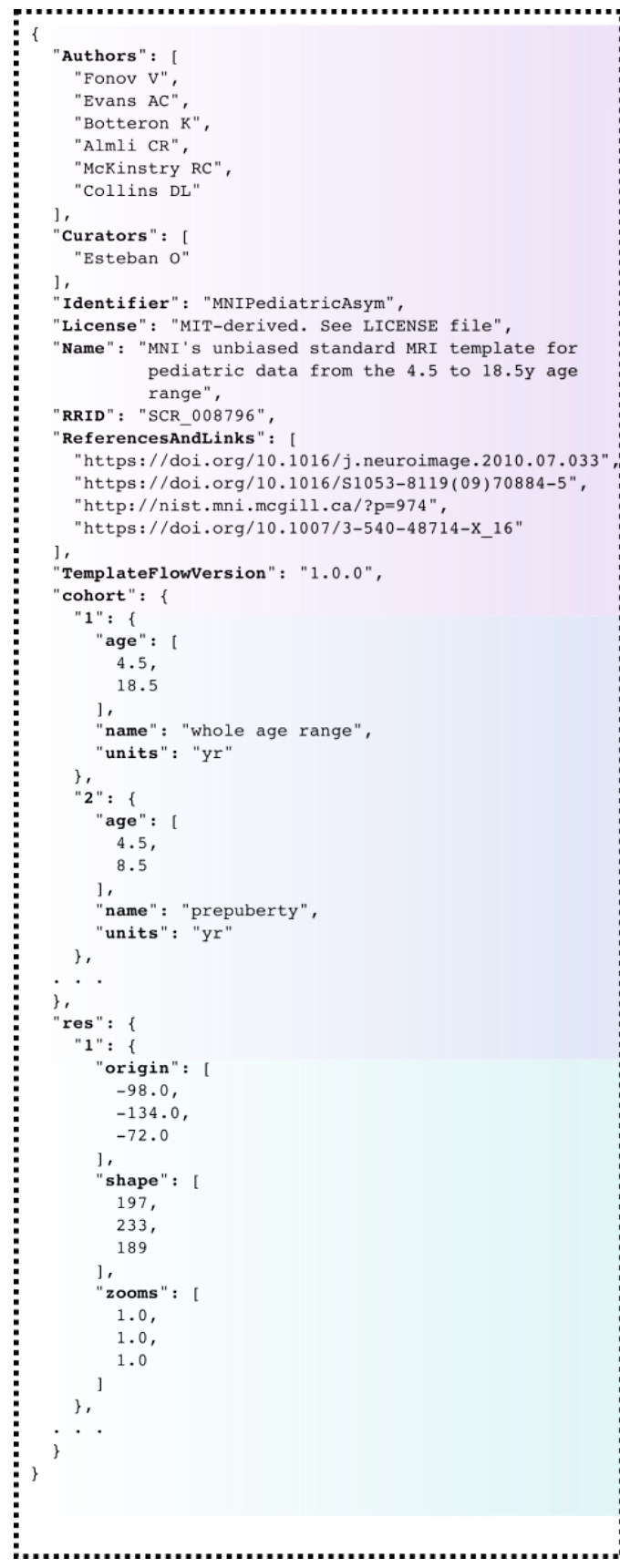

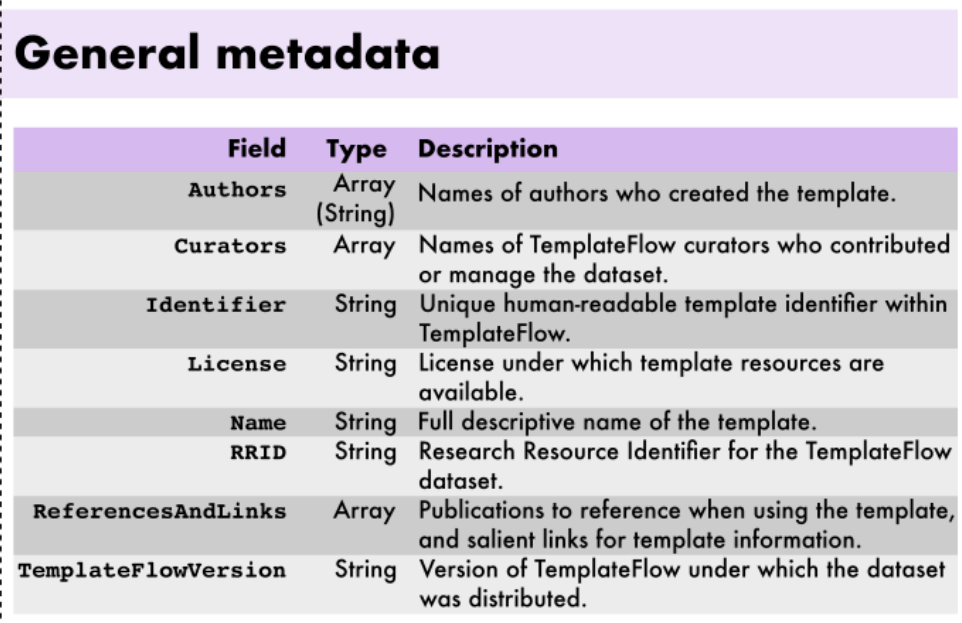

\section{Cohort metadata}

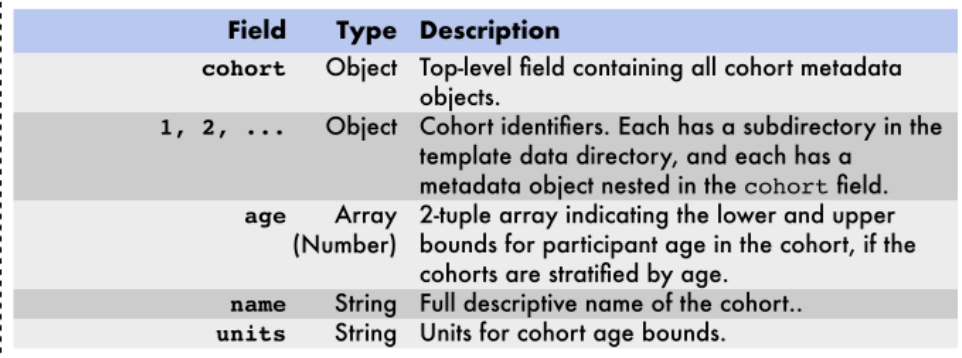

\section{Resolution metadata}

\begin{tabular}{|c|c|c|c|}
\hline & Field & Type & Description \\
\hline & res & Object & $\begin{array}{l}\text { Top-level field containing all resolution metadata } \\
\text { objects. }\end{array}$ \\
\hline & $2, \ldots$ & Object & $\begin{array}{l}\text { Resolution identifiers. Each has a metadata } \\
\text { object nested in the res field. The metadata } \\
\text { for each resolution apply to all images whose } \\
\text { name includes res-<identifier>. The identifier } \\
\text { itself does not necessarily correspond to the voxel } \\
\text { size. }\end{array}$ \\
\hline & origin & Array & $\begin{array}{l}(x, y, z) \text { spatial location of the voxel origin } \\
\text { relative to the physical origin in } \mathrm{mm} \text {. }\end{array}$ \\
\hline & shape & Array & $(x, y, z)$ shape of the image in voxels. \\
\hline & zooms & Array & $(x, y, z)$ size of each voxel in $\mathrm{mm}$ \\
\hline
\end{tabular}

Figure 5. Overview of TEMPLATEFLOW metadata specification. TEMPLATEFLOW metadata are formatted as JavaScript Object Notation (JSON) files located in each TEMPLATEFLow dataset. An example template_description.json metadata file is displayed at left (for the pediatric MNI template). In addition to general template metadata, TEMPLATEFLOW datasets can contain cohort-level and resolution-level metadata, which are nested within the main metadata dictionary and apply only to subsets of images in the dataset.

We demonstrate benefits of centralising templates in general, and the validity of the TEMPLATEFLow framework in particular, via its integration into IMRIPrep $^{38}$, a functional MRI preprocessing tool. This integration provides fMRIPrep users with flexibility to spatially normalize 
their data to any template available in the ARCHIVE. This integration has also enabled the development of fMRIPrep adaptations, for instance to pediatric populations or rodent imaging, utilizing suitable templates from the archive. The uniform interface provided by the BIDS-like directory organisation and metadata enables straightforward integration of new templates into workflows equipped to use TEMPLATEFLow templates.

TEMPLATEFLow also makes use of standards of practice from the software engineering industry, leveraging continuous delivery $(\mathrm{CD})$ and continuous integration $(\mathrm{Cl})$ tools to automate backup and synchronisation of data across projects in the templateflow organisation on GitHub. $\mathrm{Cl}$ and CD keep the web-based archive browser up to date by automatically indexing data files.
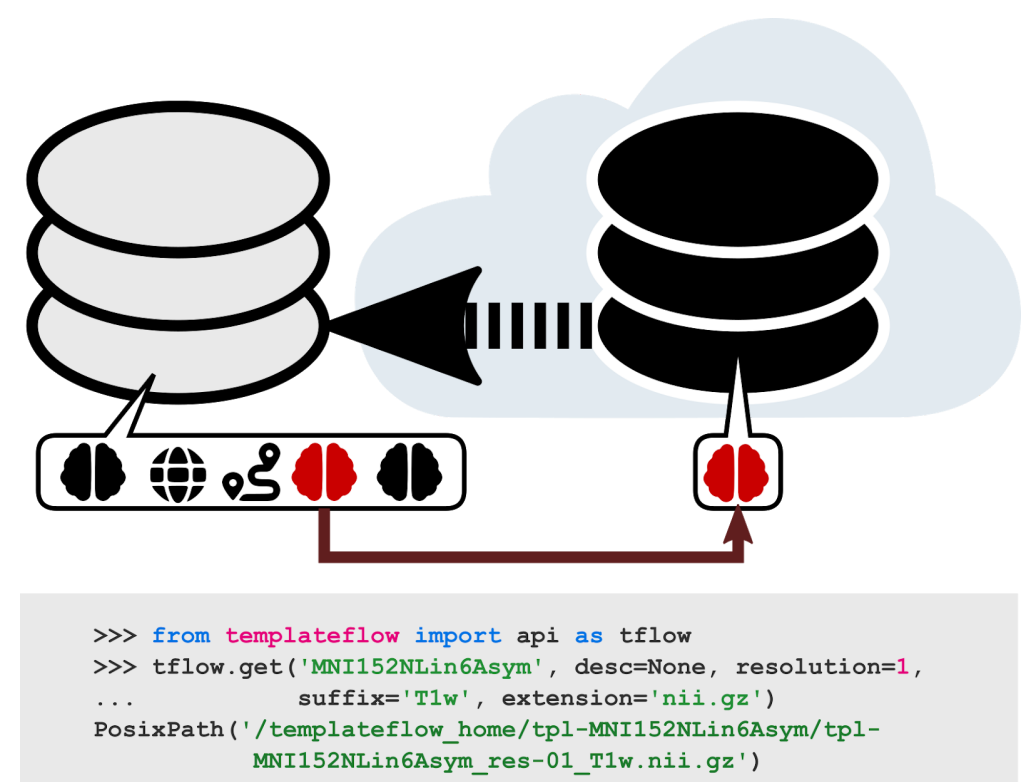

Figure 6. Example usage of the Python-based TEMPLATEFLow client. After importing the API, the user submits a query for the T1-weighted FSL version of the MNI template at $1 \mathrm{~mm}$ resolution. The client first filters through the archive, identifies any files that match the query, and finds their counterparts in cloud storage. It then downloads the requested files and returns their paths in the local TEMPLATEFLow installation directory. Future queries for the same resource can be completed without any re-downloading.

\section{Community-driven, peer-reviewed contribution process.}

A centralised repository for neuroimaging templates should also address the needs of template creators, enabling peer-reviewed integration of new templates with minimal informatic overhead. Inspired by the Conda-forge community repository and the Journal of Open Source Software (JOSS), the GitHub-based TEMPLATEFLow organisation is a site for dialogue between members of the neuroimaging community and TempLATEFLow ARCHIVE curators. GitHub issues offer any community member the ability to share their needs with developers and ARCHIVE curators, for instance by identifying templates or workflow features for potential inclusion in the 
project. Pull requests provide a means for members of the community to directly contribute code or template resources to the TempLATEFLow ARchive.

This peer-reviewed contribution process is facilitated through the Python-based TemplateFlow Manager. The TemplateFlow Manager automates the work of synchronising data from a local directory to cloud storage in OSF. Furthermore, it creates a GitHub repository containing git-annex pointers that enable $D_{A T A} L_{A D}$ to download template data from cloud storage to any machine with a copy of the repository. Finally, it opens a new pull request to propose adding the newly contributed template repository into the main TEMPLATEFLow ARCHIVE (Figure 7).

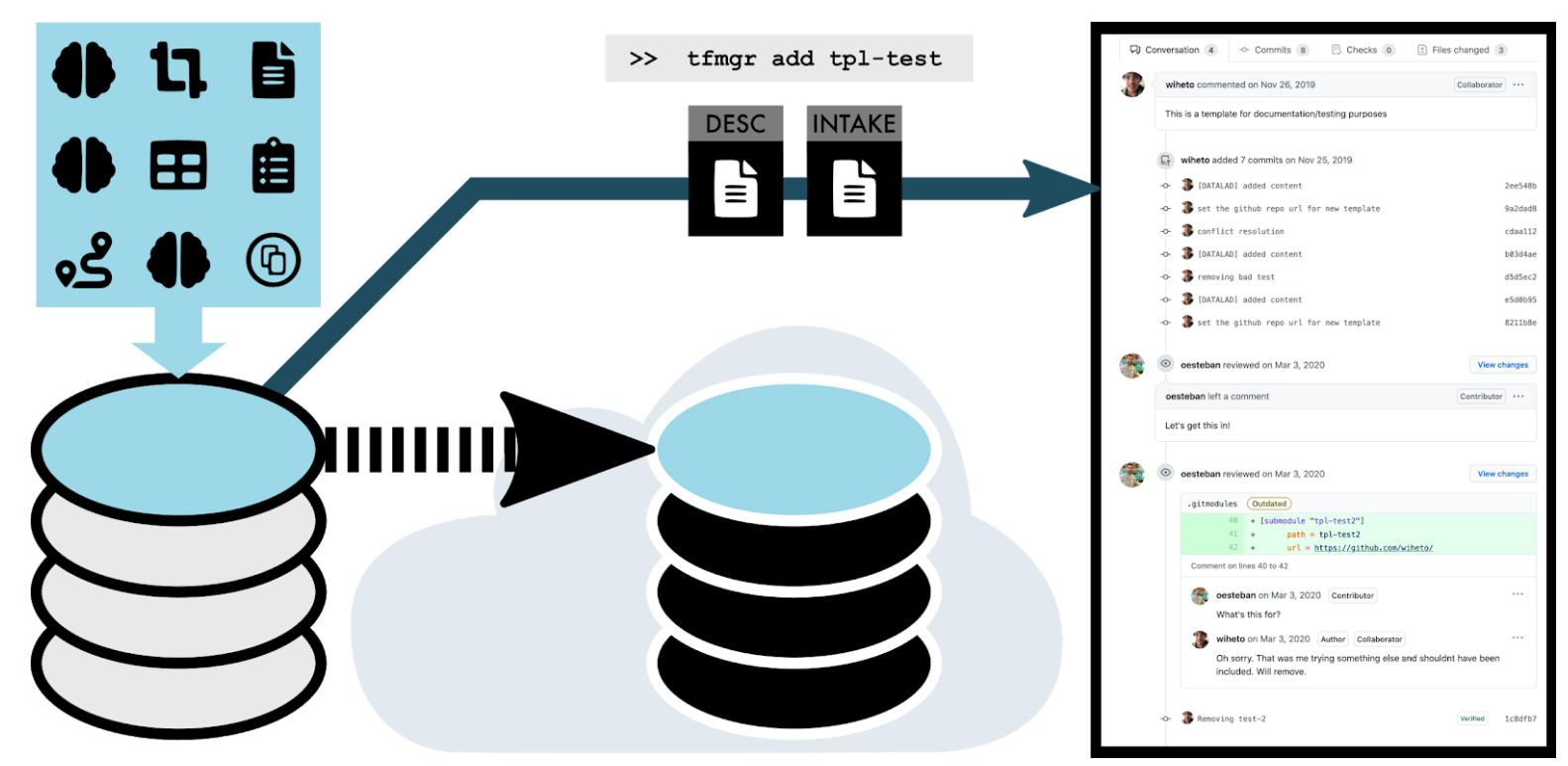

Figure 7. To contribute a new template to TEMPLATEFLOW, members of the community first organise template resources to conform to the BIDS-like TEMPLATEFLow structure. Next, tfmgr synchronises the resources to OSF cloud storage and opens a new pull request proposing the addition of the new template. A subsequent peer-review process ensures that all data are conformant with the TEMPLATEFLOW standard. Finally, TEMPLATEFLOW curators merge the pull request, thereby adding the template into the archive.

\section{Discussion}

The use of templates in neuroimaging is ubiquitous, and the emerging challenges regarding template use accordingly merit immediate attention. In an early perspective, Van Essen identified a set of desiderata for brain templates ${ }^{36}$. Above and beyond anatomical fidelity, he called for connecting templates in a "federation of databases" with "powerful and flexible options for searching, selecting, and visualizing data". Finally, he stressed the importance of resource accessibility. TempLATEFLow provides a clear foundation for a framework that satisfies all of the aforementioned desiderata. Furthermore, the ARCHIVE supports unambiguous identification of 
resources, a programmatically extensible platform for interfacing with template data, and a starting point for future investigations of inter-template concordance and robustness.

TEMPLATEFLow addresses practical issues biasing current template usage and creation. When researchers develop a new brain template or atlas for public dissemination, there exists no standard channel or format for distributing their work. With no central repository or uniform organisational scheme, template creators are often tasked with the responsibilities of maintaining template resources and managing access on an ad hoc basis. The work resulting in a template is reviewed only at publication time; subsequent template updates can go unreviewed, and any academic consensus that emerges after publication might not be associated with the original template resources at all.

Conversely, users are confronted with a surfeit of available templates and atlases, many with unclear provenance, and with the attendant challenges of locating them and integrating them into workflows. As illustrated by our text mining analysis, software libraries can further bias template usage, and the lack of consistent nomenclature between packages (as is the case for MNI templates) introduces further ambiguity in scientific communication. Leading neuroimaging toolboxes, including FSL and SPM, are packaged with a limited set of default template resources. Such limitations lead researchers to decisions regarding the creation and use of templates determined more by convenience than scientific considerations.

Together, user-facing and developer-facing issues contribute to an environment of ambiguity. Even where best practices are known, they can be difficult to locate and follow. Awareness and discoverability of extant template resources are limited by access hurdles and the use of private, decentralised communication channels, thereby driving unnecessary proliferation of template options. Integrating a template into software requires a custom solution for every new template, increasing the burden on developers. By aggregating resources in a centralised and freely accessible archive, TEMPLATEFLow maximises exposure of the scientific community to new templates and facilitates the dissemination of new template-based knowledge.

An unambiguous, accessible, and curated template portfolio. Using a software package's default template without awareness of alternatives can yield suboptimal results with adverse effects on both reproducibility and reporting. To mitigate these difficulties, TEMPLATEFLow aggregates representative template data for populations and species of scientific interest in a centralised, community-run archive. Furthermore, TEMPLATEFLow's organisational scheme enables univocal identification of brain templates, resolving the ambiguity of references to "MNI" space and thereby facilitating experimental reproducibility. More broadly, TEMPLATEFLow defines a BIDS-like language for references to brain templates and atlases ${ }^{46}$, endowing researchers with a common vocabulary so that the results they report in future work can be traced unambiguously to specific resources and replicated precisely. The archive provides template data across a range of granularities-for instance, researchers who study developmental trajectories can use a generally representative youth template or a template specific to a narrower age range according to the objectives of their research. 
An improved platform for vetting template-based knowledge and building consensus. GitHub's pull request system and integrated peer review process provide a public forum for discussion and vetting of newly proposed templates and annotations. Each template can be treated as a versioned living document that is continually reviewed and republished as necessary. If a user questions the validity or currentness of archive resources, the platform provides an immediate channel for publicly raising and addressing concerns. The discussion and vetting process forms a record of outstanding issues and concerns with existing resources that can inform researchers about the strengths and limitations of resources available to them. Ultimately, the centralisation of resources and discussion can help create a scientific consensus. As new technology enables the refinement of template resources, TEMPLATEFLow's integration with GitHub's version control system provides a built-in way to update existing templates and track revisions.

An intuitive API minimises the window for human error. The TEMPLATEFLow client implements an intuitive query system that enables programmatic retrieval of template resources from the archive. Using a command-line tool or the Python Client API, scientists can easily integrate TEMPLATEFLow into their neuroimaging analysis workflows to automate access to and use of template resources. Automation of workflows further promotes reproducibility by removing potential points of inconsistent or erroneous usage. Additionally, the BIDS-like organisation scheme of TEMPLATEFLow resources facilitates integration with BIDS apps. Within fMRIPrep, for instance, the TEMPLATEFLOW API enables flexible and systematic normalisation of preprocessed images to any template space requested by the user.

Delineating "standardness" of templates and coordinate spaces. A dispute over what qualifies as a "standard" space stems in part from the limitations of a "one-size-fits-all" approach to coordinate spaces, in part from differing study objectives, and in part from the limitations of knowledge about the organisation of the brain. In particular, the substantial variability between brains manifests not only in the morphological positions of brain features, but also in the failure of some landmarks to consistently manifest in all subjects. Functionally, deep phenotyping studies $^{60}$ have similarly revealed that the subnetwork structure of individual human brains exhibits features not present in population-level averages, up to and including entire large-scale functional networks ${ }^{61}$. Furthermore, the relationships between many brain structures and their functions either are imprecisely characterised ${ }^{62}$, defy intuitive ontologies ${ }^{63,64}$, or vary among individual subjects. Such considerations call into question the "standardness" of standard spaces derived from population averages.

These challenges are doubly amplified when researchers aim to identify inter-species homologies in the architecture of the nervous system, an endeavour that might ultimately require defining an abstract relational space that leverages spatial geometry ${ }^{65,66}$ rather than one tethered to an explicit, population-average template. The scope of the present work does not immediately encompass such challenges, but we hope that the resource introduced here can provide a starting point for future work in these veins. As registration frameworks and standard space definitions expand beyond anatomical averages and incorporate information from 
additional modalities ${ }^{67,68}$, we intend TEMPLATEFLow to grow to accommodate these new types of resources.

\section{Acknowledgements}

The development of this resource was supported by the Laura and John Arnold Foundation (RAP and KJG), the NIBIB (R01EB020740, SSG; 1P41EB019936-01A1 SSG, YOH), NIMH (R24MH114705 and R24MH117179, RAP), NINDS (U01NS103780, RAP), and NSF (CRCNS 1912266, YOH). RL is funded by the Wellcome Trust (209139/Z/17/Z). EM was supported by the UK Medical Research Council (MR/N013700/1) and King's College London. OE acknowledges financial support from the SNSF Ambizione project "Uncovering the interplay of structure, function, and dynamics of brain connectivity using MRl" (grant number PZ00P2_185872).

\section{Ethical compliance}

We complied with all relevant ethical regulations. This resource reused publicly available data derived from studies acquired at many different institutions. Protocols for all of the original studies were approved by the corresponding ethical boards.

\section{Code \& data availability statement}

All the software components discussed in this paper are available under the Apache 2.0 license, accessible as repositories of https://github.com/templateflow.

All templates and associated data are available under corresponding open licenses and accessible as described in the manuscript.

\section{Author contributions}

Conceptualization: RC, CJM, KJG, RAP, OE

Data curation: RC, EM, OE

Topics analysis: $\mathrm{RC}, \mathrm{RL}$

Funding acquisition: KJG, RAP, OE

Methodology: RC, OE

Project administration: RAP, OE

Resources: YOH, SSG, KJG, RAP, OE

Software \& Documentation: RC, WHT, MG, EM, CJM, YOH, OE

Supervision: RAP, OE

Validation: RC, WHT, EM, OE

Visualization: RC, RL.

Writing - original draft: RC \& OE

Writing - review \& editing: RC, RL, WHT, MG, EM, CJM, YOH, SSG, KJG, RAP, OE. 


\section{Online Methods}

MNI space text mining analysis. To investigate the use of the term "MNI" in the neuroimaging literature, we conducted an exploratory text mining analysis. For this, we used the Elsevier API to download the entire corpus of two leading journals of neuroimaging methodology, Neurolmage and Neurolmage:Clinical. In this way, we retrieved a total 16,812 full-text articles that were subsequently segmented into lists of sentences. A scan of these sentences revealed 14,870 sentences across 6,048 articles that contained the word "MNI". Sentences were cleaned (i.e., removing punctuation, single letters, accents, numbers) and tokenized into words, which were subsequently lemmatized (i.e., converted to base form) using the NLTK wordnet lemmatizer. From the lemmatized words, we filtered out stopwords (i.e., NLTK stopwords and a custom list) and included words with a frequency above 10 as part of our "dictionary"; this yielded a dictionary size of 2,340 words.

Next, we computed a sparse dictionary by article count matrix (i.e., 2,340 x 6,048), on which we performed topic modelling with latent Dirichlet allocation ${ }^{43}$ (LDA; implementation from scikit-learn with the learning decay hyperparameter set to 0.7$)$. The number of topics $(k=12)$ was selected by identifying the LDA model yielding the lowest perplexity ${ }^{43}$. The 20 words from the dictionary that loaded the highest on the 12 topics were visualized using word clouds. In addition, for each article we identified the most dominant topic and plotted the distribution of topics across articles.

Design and architecture. TEMPLATEFLow comprises 4 cardinal components: (i) a cloud-based archive, (ii) a Python client for programmatically querying the archive, (iii) automated systems for synchronising and updating archive data, and (iv) inter-template registration workflows. Here, we discuss the details of each component's implementation in turn, as well as the manner in which they interact with one another to form a cohesive whole.

The TemplateFlow ARchIVE. The archive itself comprises directories of template data in cloud storage. The data are stored on Google Cloud using the Open Science Framework (OSF) and on Amazon's Simple Storage Service (S3). Prior to storage, all template data must be named and organised in directories conformant to a data structure inspired by and compatible with the Brain Imaging Data Structure (BIDS) standard ${ }^{46}$. The precise implementation of this data structure is a living document and is detailed on the TEMPLATEFLow homepage (http://www.templateflow.org). We detail several critical features here.

The archive is organised hierarchically, and descriptive metadata follow a principle of inheritance: any metadata that apply to a particular level of the archive also apply to all inferior levels. At the top level of the hierarchy are directories corresponding to each archived template. If applicable, within each template directory are directories corresponding to sub-cohort templates. Names of directories and resource files constitute a hierarchically ordered series of key-value pairs terminated by a suffix denoting the datatype. For instance, tpl-MNIPediatricAsym_cohort-3_res-high_T1w.nii.gz denotes a T1-weighted template image file for resolution "high" of cohort 3 in the MNIPediatricAsym template 
(where the definitions of each resolution and cohort are specified in the template metadata file, template_description.json). The most common TempLateFlow datatypes are indexed in Table 1 of the main manuscript; an exhaustive list is available in the most current version of the BIDS standard (https://bids.neuroimaging.io/).

Within each directory, template resources include image data, atlas and template metadata, transform files, licenses, and curation scripts. All image data are stored in gzipped NIfTI format and are conformed to RAS+ orientation (i.e., left-to-right, posterior-to-anterior, inferior-to-superior, with the affine qform and sform matrices corresponding to a cardinal basis scaled to the resolution of the image). Template metadata are stored in a JavaScript Object Notation (JSON) file called template_description.json; an overview of metadata specifications is provided in Figure 5 of the main manuscript. In brief, template metadata files contain general template metadata (e.g., authors and curators, references), cohort-specific metadata (e.g., ages of subjects included in each cohort), and resolution-specific metadata (e.g., dimensions of images associated with each resolution). Atlas metadata are often stored in TSV format and specify the region name corresponding to each atlas label. Transform files are stored in HDF5 format and are generated as a diffeomorphic composition of ITK-formatted transforms mapping between each pair of templates.

The archive has a number of client-facing access points to facilitate browsing of resources. Key among these is the archive browser on the TEMPLATEFLow homepage, which indexes all archived resources and provides a means for researchers to take inventory of possible templates to use for their study.

The Python client. TemplateFlow is distributed with a Python client that can submit queries to the archive and download any resources as they are requested by a user or program. Valid query options correspond approximately to BIDS key-value pairs and datatypes. A compendium of common query arguments is provided in Table 1 of the main manuscript, and comprehensive documentation is available on the TEMPLATEFLow homepage.

When a query is submitted to the TempLateFLow client, the client begins by identifying any files in the archive that match the query. To do so, it uses PyBIDS ${ }^{58}$, which exploits the BIDS-like architecture of the TEMPLATEFLow ARCHIVE to efficiently scan all directories and filter any matching files. Next, the client assesses whether queried files exist as data in local storage. When a user locally installs TEMPLATEFLow, the local installation initially contains only lightweight pointers to files in OSF cloud storage. These pointers are implemented using $\mathrm{D}_{\mathrm{ATAL} A \mathrm{AD}^{47}}$, a data management tool that extends git and git-annex. TempLateFlow uses DataLAD principally to synchronise datasets across machines and to perform version control by tracking updates made to a dataset.

If the queried files are not yet synchronised locally (i.e., they exist only as pointers to their counterparts in the cloud), the client instructs $D_{A T A} L_{A D}$ to retrieve them from cloud storage. In the event that $D_{A T A L A D}$ fails or returns an error, the client falls back on redundancy in storage and downloads the file directly from Amazon's S3. When the client is next queried for the same file, it will detect that the file has already been cached in the local filesystem. The use of resource pointers with the client thus enables lazy loading of template resources. Finally, the client confirms that the file has been downloaded successfully. If the client detects a successful 
download, it returns the result of the query; in the event that it detects a synchronisation failure, it displays a warning for each queried file that encountered a failure.

Continued functionality and operability of the client is ensured through an emphasis on maximising code coverage with unit tests. Updating the client requires successful completion of all unit tests, which are automatically executed by continuous integration $(\mathrm{Cl})$ and continuous delivery (CD) services connected to GitHub.

Ancillary and managerial systems. TEMPLATEFLow includes a number of additional systems and programs that serve to automate stages of the archive update process, for instance addition of a new template or revision of current template resources. To facilitate the update and extension process, TEMPLATEFLow uses GitHub actions to automatically synchronise dataset information so that all references remain up-to-date with the current dataset. These actions are triggered whenever a pull request to TEMPLATEFLow is accepted. For example, GitHub actions are used to update the TEMPLATEFLow archive browser so that it displays all template resources as they are uploaded to the archive.

Whereas the TemplateFlow client synchronises data from cloud storage to the local filesystem, a complementary TEMPLATEFLow manager handles the automated synchronisation of data from the local filesystem to cloud storage. The Python-based manager is also used for template intake, i.e., to propose the addition of new templates to the archive. To propose adding a new template, a user first runs the TempLAteFlow manager using the $t f m g r$ add <template_id> - -osf-project <project_id>command.

The manager begins by using the TEMPLATEFLow client to query the archive and verify that the proposed template does not already exist. After verifying that the proposed template is new, the manager synchronises all specified template resources to OSF cloud storage. It then creates a fork of the tpl-intake branch of the TempLATEFLow GitHub repository and generates an intake file in Tom's Obvious Minimal Language (TOML) markup format; this intake file contains a reference to the OSF project where the manager has stored template resources. The TemplateFlow MANAGER commits the TOML intake file to the fork and pushes to the user's GitHub account. Finally, it retrieves template metadata from template_description.json and uses the metadata to compose a pull request on the tpl-intake branch. This pull request provides a venue for discussion and vetting of the proposed addition of a new template.

Inter-template registration workflow. To effect the flow of knowledge across template spaces, TEMPLATEFLow includes a workflow for computing robust transformations between any pair of adult human template spaces. To compute a transformation between two template spaces, the inter-template registration workflow makes use of 10 of the high-quality T1-weighted adult human brain images used in the creation of the MNI 152 template portfolio. In the first step of the workflow, these 10 images are registered to both template spaces using the symmetric normalization (SyN) algorithm ${ }^{25}$. Next, a 10-channel registration is performed in ANTs using the SyN algorithm. Thus, the workflow computes a single transformation that simultaneously optimises the alignment between all 10 images in both coordinate spaces. 


\section{References}

1. Lüders, E., Steinmetz, H. \& Jäncke, L. Brain size and grey matter volume in the healthy human brain. NeuroReport 13, 2371-2374 (2002).

2. Tosun, D., Siddarth, P., Levitt, J. \& Caplan, R. Cortical thickness and sulcal depth: insights on development and psychopathology in paediatric epilepsy. BJPsych Open 1, 129-135 (2015).

3. Tavor, I. et al. Task-free MRI predicts individual differences in brain activity during task performance. Science 352, 216-220 (2016).

4. Mars, R. B., Passingham, R. E. \& Jbabdi, S. Connectivity Fingerprints: From Areal Descriptions to Abstract Spaces. Trends Cogn. Sci. 22, 1026-1037 (2018).

5. Sowell, E. R. et al. Mapping cortical change across the human life span. Nat. Neurosci. 6, 309-315 (2003).

6. Good, C. D. et al. A voxel-based morphometric study of ageing in 465 normal adult human brains. Neurolmage 14, 21-36 (2001).

7. Courchesne, E. et al. Normal Brain Development and Aging: Quantitative Analysis at in Vivo MR Imaging in Healthy Volunteers. Radiology 216, 672-682 (2000).

8. Brett, M., Johnsrude, I. S. \& Owen, A. M. The problem of functional localization in the human brain. Nat. Rev. Neurosci. 3, 243-249 (2002).

9. von Economo, C. F., Koskinas, G. N. \& Triarhou, L. C. Atlas of cytoarchitectonics of the adult human cerebral cortex. vol. 10 (Karger Basel, 2008).

10. Brodmann, K. Brodmann's: Localisation in the Cerebral Cortex. (Springer US, 2006). doi:10.1007/b138298.

11. Schurr, P. H. \& Merrington, W. R. The Horsley-Clarke stereotaxic apparatus. Br. J. Surg. 65, 33-36 (1978).

12. Talairach, J., David, M., Tournoux, P., Corredor, H. \& Kvasina, J. Atlas d'anatomie stéréotaxique: repérage radiologique indirect des noyaux gris centraux des régions mésencéphalo-sous-optique et hypothalamique de l'homme. (Masson, 1957).

13. Talairach, J. \& Tournoux, P. Co-planar stereotaxic atlas of the human brain. (Georg Thieme Verlag/Thieme Medical Publishers, 1988).

14. Avants, B. B. et al. A reproducible evaluation of ANTs similarity metric performance in brain image registration. Neurolmage 54, 2033-44 (2011).

15. Paxinos, G. \& Watson, C. The rat brain in stereotaxic coordinates. (Elsevier Academic Press, 1997).

16. Martin, R. F. \& Bowden, D. M. Primate Brain Maps: Structure of the Macaque Brain. (Elsevier, 2000).

17. Dickie, D. A., Job, D. E., Gonzalez, D. R., Shenkin, S. D. \& Wardlaw, J. M. Use of Brain MRI Atlases to Determine Boundaries of Age-Related Pathology: The Importance of Statistical Method. PloS One 10, e0127939 (2015).

18. Matsuzawa, J. et al. Age-related Volumetric Changes of Brain Gray and White Matter in Healthy Infants and Children. Cereb. Cortex 11, 335-342 (2001).

19. Fonov, V. et al. Unbiased average age-appropriate atlases for pediatric studies. Neurolmage 54, 313-327 (2011).

20. Shi, F. et al. Infant Brain Atlases from Neonates to 1- and 2-Year-Olds. PloS One 6, 1-11 (2011).

21. Buckner, R. L. et al. A unified approach for morphometric and functional data analysis in young, old, and demented adults using automated atlas-based head size normalization: reliability and validation against manual measurement of total intracranial volume. Neurolmage 23, 724-738 (2004).

22. Calabrese, E., Badea, A., Watson, C. \& Johnson, G. A. A quantitative magnetic resonance histology atlas of postnatal rat brain development with regional estimates of growth and variability. Neurolmage 71, 196-206 (2013).

23. Szulc, K. U. et al. 4D MEMRI atlas of neonatal FVB/N mouse brain development. Neurolmage 118, 49-62 (2015).

24. Evans, A. C., Janke, A. L., Collins, D. L. \& Baillet, S. Brain templates and atlases. Neurolmage 62, 911-922 (2012). 
25. Avants, B. B., Epstein, C. L., Grossman, M. \& Gee, J. C. Symmetric diffeomorphic image registration with cross-correlation: Evaluating automated labeling of elderly and neurodegenerative brain. Med. Image Anal. 12, 26-41 (2008).

26. Balakrishnan, G., Zhao, A., Sabuncu, M. R., Guttag, J. \& Dalca, A. V. VoxelMorph: A Learning Framework for Deformable Medical Image Registration. IEEE Trans. Med. Imaging 38, 1788-1800 (2019).

27. Yoon, U., Fonov, V. S., Perusse, D. \& Evans, A. C. The effect of template choice on morphometric analysis of pediatric brain data. Neurolmage 45, 769-777 (2009).

28. Dickie, D. A. et al. Whole Brain Magnetic Resonance Image Atlases: A Systematic Review of Existing Atlases and Caveats for Use in Population Imaging. Front. Neuroinformatics 11, (2017).

29. Yeo, B. T. et al. The organization of the human cerebral cortex estimated by intrinsic functional connectivity. J. Neurophysiol. 106, 1125-1165 (2011).

30. Botvinik-Nezer, R. et al. Variability in the analysis of a single neuroimaging dataset by many teams. Nature 582, 84-88 (2020).

31. Carp, J. On the Plurality of (Methodological) Worlds: Estimating the Analytic Flexibility of fMRI Experiments. Front. Neurosci. 6, (2012).

32. Bohland, J. W., Bokil, H., Allen, C. B. \& Mitra, P. P. The Brain Atlas Concordance Problem: Quantitative Comparison of Anatomical Parcellations. PloS One 4, e7200 (2009).

33. Carp, J. The secret lives of experiments: Methods reporting in the fMRI literature. Neurolmage 63, 289-300 (2012).

34. Mazziotta, J. C., Toga, A. W., Evans, A., Fox, P. \& Lancaster, J. A Probabilistic Atlas of the Human Brain: Theory and Rationale for Its Development: The International Consortium for Brain Mapping (ICBM). Neurolmage 2, 89-101 (1995).

35. Jenkinson, M., Beckmann, C. F., Behrens, T. E. J., Woolrich, M. W. \& Smith, S. M. FSL. Neurolmage 62, 782-790 (2012).

36. Van Essen, D. C. Windows on the brain: the emerging role of atlases and databases in neuroscience. Curr. Opin. Neurobiol. 12, 574-579 (2002).

37. Esteban, O. et al. MRIQC: Advancing the Automatic Prediction of Image Quality in MRI from Unseen Sites. PloS One 12, e0184661 (2017).

38. Esteban, O. et al. fMRIPrep: a robust preprocessing pipeline for functional MRI. Nat. Methods 16, 111-116 (2019).

39. Mazziotta, J. et al. A Four-Dimensional Probabilistic Atlas of the Human Brain. J. Am. Med. Inform. Assoc. 8, 401-430 (2001).

40. Collins, D. L., Zijdenbos, A. P., Baaré, W. F. C. \& Evans, A. C. ANIMAL+INSECT: Improved Cortical Structure Segmentation. in Information Processing in Medical Imaging. 210-223 (Springer, 1999). doi:10.1007/3-540-48714-X_16.

41. Evans, A. C. et al. 3D statistical neuroanatomical models from $305 \mathrm{MRI}$ volumes. in IEEE Conference Record Nuclear Science Symposium and Medical Imaging Conference vol. 3 1813-1817 (1993).

42. Holmes, C. J. et al. Enhancement of MR Images Using Registration for Signal Averaging. J. Comput. Assist. Tomogr. 22, 324-333 (1998).

43. Blei, D. M., Ng, A. Y. \& Jordan, M. I. Latent Dirichlet Allocation. J. Mach. Learn. Res. 3, 993-1022 (2003).

44. Friston, K. J., Ashburner, J., Kiebel, S. J., Nichols, T. E. \& Penny, W. D. Statistical parametric mapping : the analysis of functional brain images. (Academic Press, 2006).

45. Bandrowski, A. E. \& Martone, M. E. RRIDs: A Simple Step toward Improving Reproducibility through Rigor and Transparency of Experimental Methods. Neuron 90, 434-436 (2016).

46. Gorgolewski, K. J. et al. The brain imaging data structure, a format for organizing and describing outputs of neuroimaging experiments. Sci. Data 3, 160044 (2016).

47. Hanke, M. et al. Open Source Software: DataLad. Zenodo (2021) doi:10.5281/zenodo.808846.

48. Nooner, K. B. et al. The NKI-Rockland Sample: A Model for Accelerating the Pace of Discovery Science in Psychiatry. Front. Neurosci. 6, (2012).

49. Marcus, D. S. et al. Open Access Series of Imaging Studies (OASIS): Cross-sectional MRI Data in Young, Middle Aged, Nondemented, and Demented Older Adults. J. Cogn. Neurosci. 19, 1498-1507 
(2007).

50. Satterthwaite, T. D. et al. The Philadelphia Neurodevelopmental Cohort: A publicly available resource for the study of normal and abnormal brain development in youth. Neurolmage 124, 1115-1119 (2016).

51. Kjonigsen, L. J., Lillehaug, S., Bjaalie, J. G., Witter, M. P. \& Leergaard, T. B. Waxholm Space atlas of the rat brain hippocampal region: Three-dimensional delineations based on magnetic resonance and diffusion tensor imaging. Neurolmage 108, 441-449 (2015).

52. Osen, K. K., Imad, J., Wennberg, A. E., Papp, E. A. \& Leergaard, T. B. Waxholm Space atlas of the rat brain auditory system: Three-dimensional delineations based on structural and diffusion tensor magnetic resonance imaging. Neurolmage 199, 38-56 (2019).

53. Papp, E. A., Leergaard, T. B., Calabrese, E., Johnson, G. A. \& Bjaalie, J. G. Waxholm Space atlas of the Sprague Dawley rat brain. Neurolmage 97, 374-386 (2014).

54. Papp, E. A., Leergaard, T. B., Calabrese, E., Allan Johnson, G. \& Bjaalie, J. G. Addendum to "Waxholm Space atlas of the Sprague Dawley rat brain" [Neurolmage 97 (2014) 374-386]. Neurolmage 105, 561-562 (2015).

55. Goerzen, D. et al. An MRI-Derived Neuroanatomical Atlas of the Fischer 344 Rat Brain. Sci. Rep. 10, 6952 (2020).

56. Van Essen, D. C., Glasser, M. F., Dierker, D. L., Harwell, J. \& Coalson, T. Parcellations and Hemispheric Asymmetries of Human Cerebral Cortex Analyzed on Surface-Based Atlases. Cereb. Cortex 22, 2241-2262 (2012).

57. Fischl, B., Sereno, M. I. \& Dale, A. M. Cortical surface-based analysis II: Inflation, flattening, and a surface-based coordinate system. Neurolmage 9, 195-207 (1999).

58. Yarkoni, T. et al. PyBIDS: Python tools for BIDS datasets. J. Open Source Softw. 4, 1294 (2019).

59. Schaefer, A. et al. Local-Global Parcellation of the Human Cerebral Cortex from Intrinsic Functional Connectivity MRI. Cereb. Cortex 28, 3095-3114 (2018).

60. Poldrack, R. A. et al. Long-term neural and physiological phenotyping of a single human. Nat. Commun. 6, 8885 (2015).

61. Laumann, T. O. et al. Functional System and Areal Organization of a Highly Sampled Individual Human Brain. Neuron 87, 657-670 (2015).

62. Cabeza, R. \& Nyberg, L. Imaging Cognition II: An Empirical Review of 275 PET and fMRI Studies. J. Cogn. Neurosci. 12, 1-47 (2000).

63. Poldrack, R. A. Mapping mental function to brain structure: How can cognitive neuroimaging succeed? Perspect. Psychol. Sci. 5, 753-761 (2010).

64. Barrett, L. F. \& Satpute, A. B. Large-scale brain networks in affective and social neuroscience: towards an integrative functional architecture of the brain. Curr. Opin. Neurobiol. 23, 361-372 (2013).

65. Stephan, K. E. \& Kötter, R. One cortex - many maps: An introduction to coordinate-independent mapping by Objective Relational Transformation (ORT). Neurocomputing 26-27, 1049-1054 (1999).

66. Huntenburg, J. M., Bazin, P.-L. \& Margulies, D. S. Large-Scale Gradients in Human Cortical Organization. Trends Cogn. Sci. 22, 21-31 (2018).

67. Robinson, E. C. et al. MSM: A new flexible framework for Multimodal Surface Matching. Neurolmage 100, 414-426 (2014).

68. Toga, A. W., Thompson, P. M., Mori, S., Amunts, K. \& Zilles, K. Towards multimodal atlases of the human brain. Nat. Rev. Neurosci. 7, 952-966 (2006).

69. Avants, B., Epstein, C., Grossman, M. \& Gee, J. Symmetric diffeomorphic image registration with cross-correlation: Evaluating automated labeling of elderly and neurodegenerative brain. Med. Image Anal. 12, 26-41 (2008).

70. Pruim, R. H. R. et al. ICA-AROMA: A robust ICA-based strategy for removing motion artifacts from fMRI data. Neurolmage 112, 267-277 (2015). 


\title{
Supplementary Materials
}

\author{
Supplementary Table 1. TEMPLATEFLow data entities.
}

\begin{tabular}{|c|c|c|}
\hline Data entity & API query example & Description \\
\hline Template & "MNI152Lin" & $\begin{array}{l}\text { The template dataset to which an image or other } \\
\text { data file belongs. }\end{array}$ \\
\hline Resolution & res $=1$ & $\begin{array}{l}\text { The image resolution. Each resolution is } \\
\text { assigned a key, which is defined in the res field } \\
\text { of template_description.json. }\end{array}$ \\
\hline Mask & $\begin{array}{l}\text { desc="brain", } \\
\text { suffix="mask" }\end{array}$ & $\begin{array}{l}\text { Indicates that the image is a binary-valued } \\
\text { annotation, where voxels labelled } 1 \text { are part of } \\
\text { the mask. }\end{array}$ \\
\hline $\begin{array}{l}\text { Discrete } \\
\text { segmentation }\end{array}$ & $\begin{array}{l}\text { desc="malf", } \\
\text { suffix="dseg" }\end{array}$ & $\begin{array}{l}\text { Indicates that the image is an integer-valued } \\
\text { annotation. Each segmentation image file } \\
\text { (.nii.gz format) is paired with a dictionary of } \\
\text { segment names (.tsv format). }\end{array}$ \\
\hline $\begin{array}{l}\text { Probabilistic } \\
\text { segmentation }\end{array}$ & $\begin{array}{l}\text { label="CSF", } \\
\text { suffix="probseg" }\end{array}$ & $\begin{array}{l}\text { Indicates that the image is a probabilistic } \\
\text { annotation, wherein the value of each voxel } \\
\text { indicates the probability of that voxel belonging to } \\
\text { the specified label. }\end{array}$ \\
\hline Atlas & $\begin{array}{l}\text { atlas="Schaefer", } \\
\text { desc="7Network" }\end{array}$ & The atlas to which a segmentation file belongs. \\
\hline Transformation & $\begin{array}{l}\text { from="MNI152Lin", } \\
\text { suffix="xfm" }\end{array}$ & $\begin{array}{l}\text { File containing a mapping between } 2 \text { stereotaxic } \\
\text { coordinate spaces. The source space is defined } \\
\text { in the from field, while the target space is } \\
\text { defined in the tpl field. }\end{array}$ \\
\hline
\end{tabular}

Image modality suffix="T1w"

For non-annotation brain images, the suffix indicates whether the image is T1-weighted ( $\mathrm{T} 1 \mathrm{w}), \mathrm{T} 2$-weighted ( $\mathrm{T} 2 \mathrm{w})$, proton density-weighted (PD), or T2*-weighted (T2star).

Template cohort $=1$ cohort

Subsample of a dataset used to generate an average template. 


\section{Supplementary Table 2. Command-line interface for TEMPLATEFLow MANAGER.}

\begin{tabular}{|c|c|c|}
\hline Argument & Environment variable & Specifications \\
\hline template_id & & $\begin{array}{l}\text { Identifier of the template. This is the value of } \\
\text { the tpl field in all file names. }\end{array}$ \\
\hline --osf_project & OSF_PROJECT & $\begin{array}{l}\text { The OSF project where the template data } \\
\text { are to be stored. The project must be } \\
\text { writable by the user account whose } \\
\text { credentials are specified in the } \\
-- \text { osf-user and--osf-password } \\
\text { arguments. }\end{array}$ \\
\hline --osf_user & OSF_USERNAME & $\begin{array}{l}\text { Account username or identifier for OSF } \\
\text { cloud storage. }\end{array}$ \\
\hline --osf_password & OSF_PASSWORD & Account password for OSF cloud storage. \\
\hline --osf_overwrite & & $\begin{array}{l}\text { Flag that indicates that the OSF client } \\
\text { should force the overwrite of any existing } \\
\text { files in the OSF project that have names } \\
\text { conflicting with those of new files. }\end{array}$ \\
\hline --gh-user & GITHUB_USER & $\begin{array}{l}\text { Account username for GitHub. The user } \\
\text { account whose credentials are provided } \\
\text { must have a fork of the TEMPLATEFLow repo. }\end{array}$ \\
\hline --gh-password & GITHUB_PASSWORD & Account password for GitHub. \\
\hline --path & & $\begin{array}{l}\text { Path to a local directory where template } \\
\text { resources are located. The path must either } \\
\text { be a directory whose name is } \\
\text { tpl-<template_id }>\text { or contain such a } \\
\text { directory. }\end{array}$ \\
\hline
\end{tabular}

- - nprocs
Maximum number of parallel processes to run when uploading to or fetching from OSF. 


\section{Supplementary Box 1. The TeMPLATEFLow MANAGER utility.}

As a reference, we provide a brief protocol for contribution of templates stored on OSF. The requirements for using this protocol are a GitHub account, the hub command-line interface for GitHub (https://github.com/github/hub), an OSF account, the OSF Python client (downloadable from https://github.com/osfclient/osfclient), the TempLAteFlow Python client (downloadable from https://github.com/templateflow/python-client), and the TEMPLATEFLow archive manager (downloadable from https://github.com/templateflow/python-manager).

1. First, locally aggregate and organise template resources in a top-level tpl directory according to the TeMPLATEFLow specification (Figure 2, Table 1). The tpl directory must use a unique name not assigned to any existing templates in the archive. It should contain template metadata in a JSON file called template_description.json (Figure 5).

2. Next, create a new project on OSF to store templates that meet the TempLateFLow specification.

3. Execute tfmgr. The minimal syntax for adding a new template is tfmgr add <template_id> --osf-project <project_id>. Arguments to tfmgr are detailed in Table 3. In place of directly supplying command-line arguments, it is also possible to configure shell environment variables that will automatically populate arguments in future runs of $t \mathrm{fmg} r$. $t$ fmgr will automatically upload the resources from step 1 to the OSF project from step 2. It will then add git-annex pointers to the data to the forked repository from step 3 . Finally, it will open a pull request that proposes adding the new template resources to the main TempLateFlow ARchive. 


\section{Supplementary Box 2. A framework where atlas knowledge flows across templates.}

Researchers frequently report coordinates and define new atlases with reference to the stereotaxic coordinate spaces defined by template images. To access the information encoded in those atlases and coordinates, scientists are accordingly required to perform their own analyses with respect to the same template image. If scientists then wish to access information defined with reference to another template, they must perform further, potentially costly analytic steps.

We designed TemplateFlow to facilitate the transfer of information across the coordinate spaces defined by templates. To fulfill this end, a standardised and version-controlled archive of template datasets must be complemented with a means of mapping features between template images. These mappings take the form of bi-directional, diffeomorphic transformation functions that can be composed to project data from any template to any other template. Accordingly, TEMPLATEFLow incorporates an image registration workflow that leverages the top-performing symmetric image normalisation (SyN) algorithm from ANTs to compute template-to-template transforms between human brain template datasets ${ }^{69}$.

The TEMPLATEFLow registration framework proceeds in several steps (Supplementary Figure 1). First, a sample of 10 high-quality ${ }^{37}$ adult human T1-weighted images from the MNI 152 database are subject to atlas-based brain extraction (or skull-stripping), which sets all non-brain voxels to zero. The 10 skull-stripped images are then aligned via SyN to the two templates between which the transformation is being computed, thus obtaining maps of all 10 images transformed into both templates' coordinate spaces. Afterward, a single, high-quality template-to-template transform is obtained by computing a 10-channel registration to simultaneously maximise the alignment of all 10 images from one template's coordinate space into the other's. 


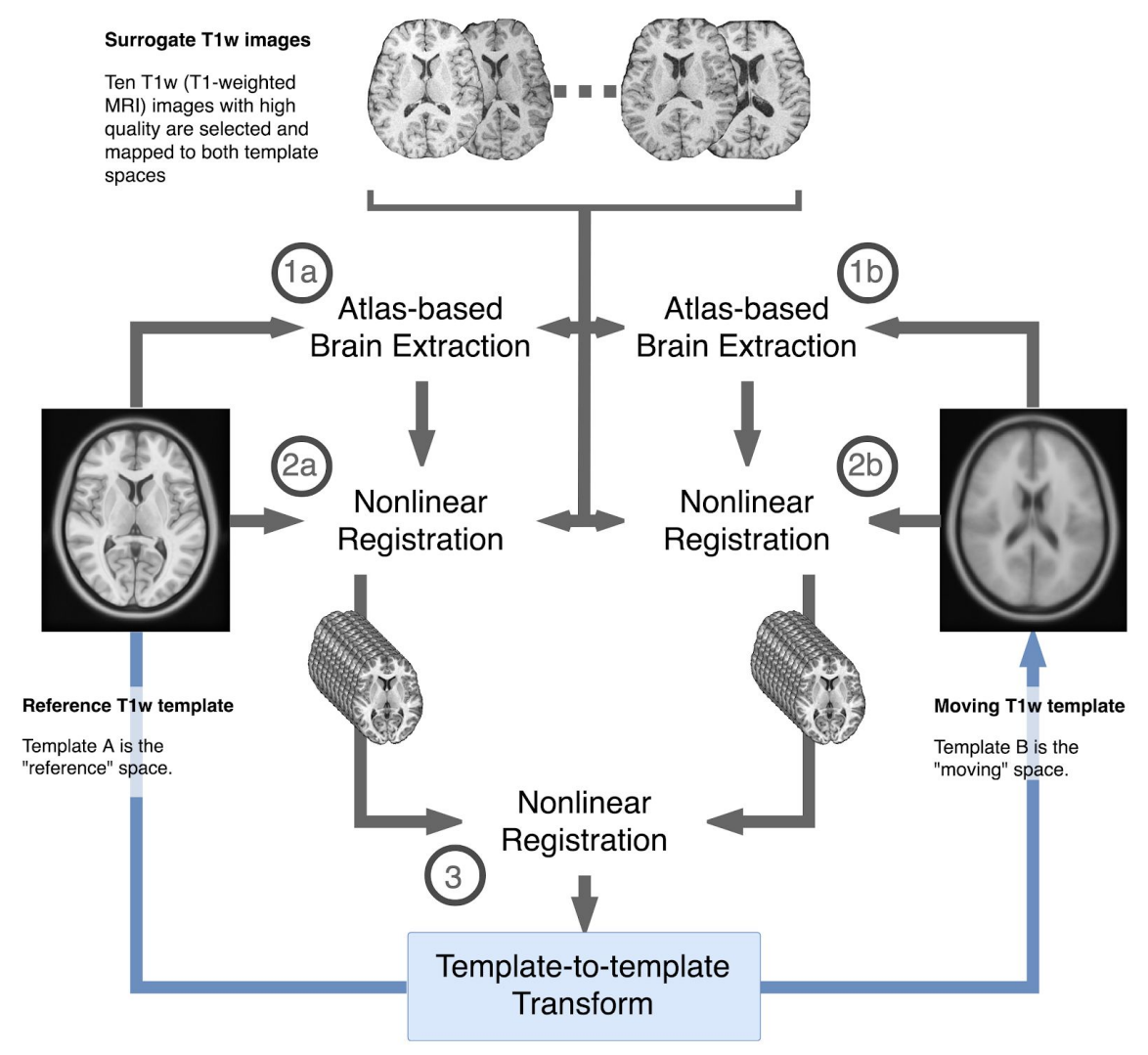

Supplementary Figure 1. The TEMPLATEFLOW registration framework. To share information between human brain templates, TEMPLATEFLOW computes a complete set of transformations that map between all human template datasets in the TEMPLATEFLow ARCHIVE. Each template-to-template transformation is computed as follows: first, 10 T1 images are skull-stripped and then registered to both templates. A single registration is then computed between the two template spaces so as to simultaneously optimise the alignment between all 10 images in both spaces.

Each template-to-template transform is a function that maps images from a source template space into a target template space. The TEMPLATEFLow archival specification pairs each transform with an intuitive name that allows users to easily determine the transform's source and target. Specifically, TeMPLATEFLow archives template-to-template transforms in the directories of their target templates. Transforms are stored in HDF5 format and are denoted with the suffix $\times f m$, and their source templates are denoted in the from field of the transform file name. Transforms can be readily applied to datasets using ANTs, supporting the sharing of atlas knowledge across template datasets.

By chaining template-to-template transforms with the template-to-subject transforms typically computed in the course of a standard neuroimaging workflow, atlas knowledge can also freely flow from any TEMPLATEFLow dataset into subject-specific coordinate spaces. 
Supplementary Box 3. Example use case: ICA-AROMA.

Suppose that a user wishes to denoise a BOLD MR image using ICA-AROMA ${ }^{70}$ and then perform regional analyses using atlases defined with respect to the most recent MNI152NLin2009cAsym template ${ }^{19,40}$. Because ICA-AROMA operates in FSL's MNI152NLin6Asym space ${ }^{24}$, this workflow would be nontrivial to configure, and inaccurate configuration would incur analytic inconsistencies. Further complications arise if the target population is not neurotypical human adults. TEMPLATEFLow undergirds fMRIPrep's solution to this problem by providing a uniform system for retrieving resources that allow both templates to be integrated into the processing workflow ${ }^{38}$. In particular, the same code can be reused to register the functional image to both target templates. ICA decomposition and identification of motion-related components by way of the ICA-AROMA algorithm are performed in the MNI152NLin6Asym space. The time series of components identified as noise can then be filtered in the subject's native space before fMRIPrep projects the BOLD data into all desired target spaces, including MNI152NLin2009cAsym.

Ease of use and broad access are not without attendant challenges. The friction preventing inappropriate use of resources is arguably reduced when a wider assortment of resources is on hand, and users are afforded additional liberty and responsibility for selecting the resource most appropriate for their objective. For instance, there is no inherent mechanism preventing a researcher from using an adult template for a study of infants, although this usage would not be well-motivated. Thus, TEMPLATEFLow resources must be used with active intent and deliberation on the part of the user. 